\title{
GENERAL BOUNDARY VALUE PROBLEMS FOR ELLIPTIC PARTIAL DIFFERENTIAL EQUATIONS
}

\author{
BY MARTIN SCHECHTER
}

Communicated by Lipman Bers, November 19, 1958

In this paper we combine the methods of Aronszajn and Milgram [3] with those previously employed by the author [9] and solve very general boundary value problems for elliptic equations. For convenience we consider equations, but much of what we say can be carried over to systems without difficulty.

Let $\mu=\left(\mu_{1}, \mu_{2}, \cdots, \mu_{n}\right)$ be a sequence of indices, each $\geqq 0$, and set

$$
\begin{aligned}
|\mu| & =\sum_{\mid \mu k} \mu_{k}, \quad \xi^{\mu}=\xi_{1}^{\mu_{1} \mu_{2}^{\mu_{2}}} \cdots \xi_{n}^{\mu_{n}}, \\
D^{\mu} & =\partial^{|\mu|} /\left(i \partial x_{1}\right)^{\mu_{1}}\left(i \partial x_{2}\right)^{\mu_{2}} \cdots\left(i \partial \chi_{n}\right)^{\mu_{n}}
\end{aligned}
$$

where $\xi=\left(\xi_{1}, \xi_{2}, \cdots, \xi_{n}\right)$ is any $n$-dimensional vector. The linear partial differential operator of order $2 r$

$$
A=\sum_{|\mu| \leqq 2 r} a_{\mu}(x) D^{\mu}
$$

with complex coefficients $a_{\mu}(x)$ is elliptic in a region $R \subseteq E^{n}$ if its characteristic polynomial

$$
P(x, \xi)=\sum_{|\mu|=2 r} a_{\mu}(x) \xi^{\mu}
$$

does not vanish in $R$ for real $\xi \neq 0$. If $R$ is the closure $\bar{G}$ of a bounded domain $G$, we shall say that $A$ is properly elliptic in $\bar{G}$ if in addition it satisfies at every point $x$ on the boundary $\dot{G}$ of $G$ (cf. $[2 ; 5 ; 8])$.

Condition 1 . For every real vector $\tau \neq 0$ parallel to $\dot{G}$ at $x$ and every real $\nu \neq 0$ normal to $\dot{G}$ at $x$, the polynomial $P(z)=P(x, \tau+z \nu)$ has exactly $r$ roots $\lambda_{k}(\tau, \nu), k=1,2, \cdots, r$, with positive imaginary parts.

If $n>2$, all elliptic operators are properly elliptic.

By a boundary operator we shall mean a linear partial differential operator whose coefficients need merely be defined on $\dot{G}$. If

$$
B_{j}=\sum_{|\mu| \S m_{j}} b_{j \mu}(x) D^{\mu}, \quad j=1,2, \cdots, r,
$$

is a set of such operators, we set

$$
Q_{j}(x, \xi)=\sum_{|\mu|=m_{j}} b_{j \mu}(x) \xi^{\mu}, \quad j=1,2, \cdots, r .
$$

We shall say that the set $\left\{B_{j}\right\}_{j=1}^{r}$ "covers" a properly elliptic operator $A$ if each $m_{j}<2 r$ and the $B_{j}$ satisfy at each point $x \in \dot{G}$. 
Condition 2. For every $\tau \neq 0$ parallel to $\dot{G}$ at $x$ and every $\nu \neq 0$ normal to $\dot{G}$ at $x$ the polynomials $Q_{j}(z)=Q_{j}(x, \tau+z \nu), j=1,2, \cdots, r$, are linearly independent modulo the polynomial

$$
S(z)=\prod_{k=1}^{r}\left(z-\lambda_{k}(\tau, \nu)\right)
$$

From now on we shall assume that $G$ is bounded, $\dot{G}$ is of class $C^{\infty}$, and all functions considered are in $C^{\infty}(\bar{G})$. We set

$$
\begin{aligned}
(u, v)_{s} & =\int_{G} \sum_{|\mu| \leqq s} D^{\mu} u \overline{D^{\mu} v d x} \\
\|v\|_{s}^{2} & =(v, v)_{s}, \quad(u, v)=(u, v)_{0}
\end{aligned}
$$

and denote the formal adjoint of $A$ by $A^{*}$. The set $\left\{B_{j}\right\}_{j=1}^{r}$ is called normal if $m_{j} \neq m_{k}$ for $j \neq k$ and $Q_{j}(x, \nu) \neq 0$ for each $j$ and $x \in \dot{G}$ when $\nu \neq 0$ is normal to $\dot{G}$ at $x$.

Lemma 1 (ARonszajn-Milgram [3]). Let $A$ be an elliptic operator of order $2 r$, and let $\left\{B_{j}\right\}_{j=1}^{r}$ be a normal set of boundary operators of orders $m_{j}<2 r$, respectively. Then we can find another normal set $\left\{B_{j}^{*}\right\}_{j=1}^{r}$ such that

$$
\left(u, A^{*} v\right)=(A u, v)
$$

for all $v$ satisfying

$$
B_{j}^{*} v=0 \quad \text { on } \dot{G}, \quad j=1,2, \cdots, r \text {, }
$$

if and only if

$$
B_{j} u=0 \quad \text { on } \dot{G}, \quad j=1,2, \cdots, r .
$$

We call the set $\left\{B_{j}^{*}\right\}_{j=1}^{r}$ adjoint to $\left\{B_{j}\right\}_{j=1}^{r}$ relative to $A$. We also have

LEMMA 2. The normal set $\left\{B_{j}\right\}_{j=1}^{r}$ covers $A$ if and only if every normal set adjoint to $\left\{B_{j}\right\}_{j=1}^{r}$ relative to $A$ also covers $A$.

The boundary value problem $\pi\left(A, f, u_{0}, B_{j}\right)$ is to find a function $u \in C^{\infty}(\bar{G})$ such that $A u=f$ in $G$ and $B_{j} u=B_{j} u_{0}$ on $\dot{G}, j=1,2, \cdots, r$. We now state our main result.

Theorem. Let $A$ be a properly elliptic operator of order $2 r$ which is covered by a normal set $\left\{B_{j}\right\}_{j=1}^{r}$. Let $\left\{B_{j}^{*}\right\}_{j=1}^{r}$ be any normal set adjoint to $\left\{B_{j}\right\}_{j=1}^{r}$ relative to $A$. Then the boundary value problem $\pi\left(A, f, u_{0}, B_{j}\right)$ has a solution for every $f$ and $u_{0}$ if and only if the solution of $\pi\left(A^{*}, 0,0, B_{j}^{*}\right)$ is unique. 
Outline of Proof. Complete $C^{\infty}(\bar{G})$ with respect to the norm \|\|$_{2 r}$ and call the resulting Hilbert space $H$. For convenience we assume $u_{0}=0$. Set

$$
[u, v]=\left(A^{*} u, A^{*} v\right)+\sum_{j=1}^{r}\left\langle B_{j}^{*} u, B_{j}^{*} v\right\rangle_{2 r-m_{j}},
$$

where the $\langle,\rangle_{s}$ are appropriate boundary inner products (cf. $[1 ; 2$; 8]). By Lemma 2 it follows that the set $\left\{B_{j}^{*}\right\}_{j=1}^{r}$ covers $A$ (and consequently $\left.A^{*}\right)$. The results of $[1 ; 2 ; 8]$ and the uniqueness of $\pi\left(A^{*}, 0,0, B_{\jmath}^{*}\right)$ imply

$$
c^{-1}\|v\|_{2 r}^{2} \leqq[v, v] \leqq c\|v\|_{2 r}^{2} \quad \text { for all } v \in H .
$$

It then follows from the Lax-Milgram lemma [6] that there is a $g \in H$ such that

$$
[g, v]=(f, v) \quad \text { for all } v \in H .
$$

Applying regularity theory similar to that of Nirenberg [7] and Brow$\operatorname{der}[4]$ we then prove that $g \in C^{\infty}(\bar{G})$. Hence $u=A^{*} g$ satisfies $A u=f$ in $G$ and $\left(u, A^{*} v\right)=(A u, v)$ for all $v \in C^{\infty}(\bar{G})$ satisfying (2). Thus $u$ satisfies (3) by Lemma 1 and the proof is complete.

\section{REFERENCES}

1. Shmuel Agmon, The coerciveness problem for integro-differential forms, Technical Note No. 5, The Hebrew University, Jerusalem, 1958.

2. Shmuel Agmon, Avron Douglis, and Louis Nirenberg, Estimates near the boundary for solutions of elliptic partial differential equations satisfying general boundary conditions. I, Comm. Pure Appl. Math., to appear.

3. Nachman Aronszajn and A. N. Milgram, Differential operators on Riemannian manifolds, Rend. Circ. Mat. Palermo, vol. 2, Ser. 2, 1953, pp. 1-61.

4. F. E. Browder, On regularity properties of solutions of elliptic differential equations, Comm. Pure Appl. Math. vol. 9 (1956) pp. 351-361.

5. Lars Hörmander, On the regularity of the solutions of boundary problems, Acta Math. vol. 99 (1958) pp. 225-264.

6. P. D. Lax and A. N. Milgram, Parabolic equations, Contributions to The Theory of Partial Differential Equations, Annals of Mathematics Studies, no. 33, Princeton, 1954, pp. 167-190.

7. Louis Nirenberg, Remarks on strongly elliptic partial differential equations, Comm. Pure Appl. Math. vol. 8 (1955) pp. 648-674.

8. Martin Schechter, Integral inequalities for partial differential operators and functions satisfying general boundary conditions, Comm. Pure Appl. Math. vol. 12 no. 1 (1959).

9. - Solution of the Dirichlet problem for equations not necessarily strongly elliptic, Bull. Amer. Math. Soc. vol. 64 (1958) pp. 371-372.

New York University 